\title{
Left Ureter
}

National Cancer Institute

\section{Source}

National Cancer Institute. Left Ureter. NCI Thesaurus. Code C49324.

The ureter connecting the left kidney to the bladder. 